\title{
Las derechas políticas, el enfoque sociohistórico y algunos apuntes personales
}

\author{
Political right-wings, the socio-historical approach, and some personal remarks
}

\author{
Sergio Daniel Morresi \\ smorresi@gmail.com \\ Instituto de Humanidades y Ciencias Sociales - \\ CONICET - Universidad Nacional del \\ Litoral, , Argentina
}

Cita sugerida: Morresi, S. D. (2021). Las derechas políticas, el enfoque sociohistórico y algunos apuntes personales. Sociohistórica, 47, e131. https://doi.org/10.24215/18521606e131

\begin{abstract}
Resumen: Tomando como punto de partida algunos debates de la ciencia política, este artículo busca mostrar la utilidad de incorporar la dimensión histórica al estudio de ciertos fenómenos políticos contemporáneos. A través de la exposición de una trayectoria académica se explica cómo se arribó al enfoque de la derecha como un campo (es decir, como una configuración de relacionales sociales de competencia, colaboración y conflicto dotada de una gravedad específica, que se impone a sus propios componentes) y se pone de relieve en qué sentido la historia y el trabajo historiográfico resultan imprescindibles para analizarlo.

Palabras clave: Ciencia Política, Historia Política, Derechas Políticas, Entornos partidarios.
\end{abstract}

Abstract: This article aims to show the utility of incorporating the historical dimension into the study of certain contemporary political phenomena, taking as a starting point some debates in political science. Through the exposition of an academic trajectory, it's explained the right-wing as a field approach (that is, the right-wing as a configuration of social relations of competition, collaboration, and conflict endowed with a specific gravity imposed on its components). Additionally, the text highlights why history and historiographical work are essential to analyze the right-wing as a field.

Keywords: Political Science, Political History, Political rightwings, Party Entourages.

Estas páginas buscan pasar en limpio una serie de interesantes preguntas y respuestas que se dispararon a partir del cuestionario elaborado por el Grupo de Investigación Estado, Desarrollo y Políticas Públicas (GIDEPP-IHUCSO, CONICET/UNL), el Grupo de Estudios sobre Derecho, Historia y Cuestión Ambiental (FCJS-UNL) y el Grupo Interdisciplinario de Estudios Socio-históricos (GIESH-IHUCSO, CONICET/UNL). También son un intento por incorporar algunas de las discusiones que tuvieron lugar en el encuentro Usos de la Historia para Investigar Problemas Sociales, que estos colectivos organizaron en la ciudad de Santa Fe en 2019. En parte por ello el texto tiene un formato particular: está escrito en primera persona del singular y bascula entre la memoria personal y la académica, la opinión y el comentario argumentado. Si bien opté por corregir y adaptar la versión original que fue discutida en el encuentro santafesino, me pareció que era preferible mantener en la medida de lo posible el tono de ese primer escrito, 
porque entiendo que colabora con el objetivo que se buscaba desde el inicio: ilustrar usos de la historia que resultan no solo de diferentes agendas de investigación, sino también de distintos recorridos académicos y profesionales.

\section{Ciencia Política, teoría e historia}

Como sucedió antes con otras disciplinas, durante décadas la ciencia política se dedicó con cierta tenacidad a disputar su autonomía por medio de una celosa demarcación de lo que consideraba su incumbencia, lo político, y defendiendo una forma determinada de abordar a ese objeto. Así, por ejemplo, Giovanni Sartori $(1974,2002)$ entendía a la ciencia política como un método distinto de la teoría o la filosofía, que consistía, ante todo, en la comparación rigurosa de tangibles y observables formas de hacer, que se desarrollaban en un determinado ámbito (el del sistema político). Cuando era un estudiante de grado, en la década de 1990, esta concepción me producía cierta incomodidad. Por aquel tiempo, lo que me interesaba a mí era la teoría política, una forma de conocimiento que, aseguraban algunos, no era propiamente parte de la disciplina sino más bien de sus antecedentes. Sartori (2002) afirmaba que la teoría política era una suerte de puente entre la filosofía y la ciencia, y que estaba destinada a desaparecer, reabsorbida por una robusta y empirista ciencia de la política. Algunos especialistas llegaban a afirmar que, si Aristóteles o Marx llegaron alguna vez a producir un saber valioso, ese saber ya estaría incorporado al conocimiento científico actual y que el resto de sus ideas carecía de relevancia (excepto para los historiadores). En un manual para estudiantes, Gabriel Almond (1996, p. 51) afirmaba que "la mayoría de los colegas acuerda en que Michael Walzer tiene una mayor comprensión del concepto de Justicia que la que podía tener Platón (...) y que Robert Dahl nos brinda una mejor teoría de la democracia que Aristóteles". Josep Colomer afirmó en un sentido similar:

un signo evidente de debilidad [de la Ciencia Política] es que, a diferencia de lo que ocurre en economía y en otras ciencias sociales, en los estudios políticos todavía se siga colocando a los autores llamados "clásicos" en el mismo nivel o incluso más alto- que a los investigadores contemporáneos. Por decirlo rápido, casi ningún escrito de Machiavelli o de Montesquieu o de la mayoría de los demás habituales en la lista sagrada sería hoy aceptado para ser publicado en una revista académica internacional con evaluadores anónimos. Cualquier persona versada en leer literatura académica contemporánea que consulte los "clásicos" debería reconocer que una gran parte de sus textos son confusos y ambiguos, y por eso varias generaciones de académicos siguen dilapidando sus vidas tratando de averiguar "qué es exactamente lo que quiso decir..." hace unos años Marx, hoy quizá Nietzsche o Tocqueville (Colomer, 2006, p. 43)

Justamente eso que Colomer llamaba "dilapidar" la vida era lo que yo hacía cuando era estudiante (Morresi, 1997) , cuando cursaba mi doctorado (Morresi, 2002) y algo que -de hecho- todavía hago (Morresi, 2018) . Pero eso que para algunos carecía de sentido, para mi tenía (y tiene) valor. La teoría política es diferente a la filosofía política, que se pregunta, por ejemplo, "qué es la justicia" o "cómo debe ser una sociedad justa”. También es distinta de la historia de las ideas, preocupada por los contextos sociales o lingüísticos y las intenciones de quienes enuncian ideas. Lo propio de la teoría política, diría yo, es una búsqueda por comprender la realidad, y (en ocasiones) por ofrecer razones para cambiar esa realidad en determinado sentido. Así, un trabajo de teoría política puede discutir sobre el concepto de democracia en el sentido filosófico y puede incluir una historia de ese concepto, pero pondrá el foco sobre todo en comprender el modo en que opera la democracia y en incidir sobre ese modo. Ahora bien, una parte importante del trabajo de los que se dedican a la teoría política consiste en estudiar a los teóricos que forman el canon para comprender y cuestionar los vericuetos de sus argumentos. Incluso aquellos autores que proponen su propia perspectiva dedican tiempo y esfuerzo a estudiar a otros teóricos del presente o del pasado, discutiendo con ellos de una forma similar a aquella que Maquiavelo le contaba en su célebre carta a su amigo Francesco Vittori (Machiavelli, 1969). Obviamente, muchos de los que se dedican a la teoría política se detienen mucho antes de formular una propuesta original y se dedican a analizar a algunos autores o a una tradición, buscando incidir o intervenir en el diálogo a partir de ofrecer una nueva lectura (o incluso reposicionando lecturas y perspectivas olvidadas) de obras ya consagradas. Pero incluso esto último, lejos de ser una "pérdida de 
tiempo", como supone Colomer, es parte de la construcción de una conversación constante que difícilmente sea reabsorbida, como en su momento planteara Sartori.

Cuando comencé mi doctorado en Brasil, hace veinte años, mi idea era continuar dentro del campo de la teoría política. Mi tesis (Morresi, 2006) fue una crítica a las lecturas neoliberales del liberalismo clásico, y en ella sostuve que los autores libertarianos o neoliberales tenían una lectura sesgada, partisana y no razonable de los liberales clásicos como John Locke o Adam Smith. Mi objetivo era brindar argumentos para rechazar la idea de que existía una continuidad entre el liberalismo clásico y el neoliberalismo. Pero para hacer eso debí lidiar de un modo mucho más serio con ciertos saberes y métodos que eran propios de la historia de las ideas y de la historia intelectual (Dosse, 2007; Skinner, 2002; Pocock, 2011; Bevir, 1999). Fue por ese vértice que la historia comenzó a ser central en mi trabajo.

Ya de vuelta en Argentina, me interesé en entender cómo el neoliberalismo había logrado imponerse de un modo tan cabal en mi país. Quería comprender por qué, de qué modo, eso que yo estudiaba se había hecho hegemónico. Las preguntas que me hacía entonces entraban en distintos terrenos (la historia reciente, la sociología política y la ciencia política), pero como mi experiencia en investigación era de teoría política e historia intelectual, fue lógico que usase las herramientas que ya conocía para mi nuevo objetivo. Así, le di un lugar privilegiado a la lectura de los textos fundamentales del neoliberalismo y el libertarianismo; le presté atención a los contextos y trabajé sobre la forma en que estas ideas fueron recibidas, traducidas y adaptadas en Argentina. De este modo, me fui acercando lentamente a una de las formas de abordar el neoliberalismo que venían desarrollando otros especialistas como Dieter Plehwe y Philip Mirowski. En lugar de considerar al neoliberalismo como un conjunto de políticas determinadas (lo que entonces era un enfoque usual en el mundo académico) o un mero discurso "encubridor de intereses" (idea muy presente en las discusiones públicas argentinas), la propuesta era comprenderlo como un proyecto de un colectivo políticointelectual situado históricamente y que reconocía múltiples modulaciones (Plehwe; Walpen y Neunhöffer, 2005; Mirowski y Plehwe, 2009; Mirowski, 2014; Plehwe, 2018).

En este punto, entiendo, el uso de la historia al que me acercaba no era el recomendado por la ciencia política. Para lo que podríamos llamar las corrientes principales de la disciplina, la historia era algo a ser "usado" de un modo determinado: formular y (de modo auxiliar y débil) controlar hipótesis (Bartolini, 1993, 1994). En palabras de Sartori:

\footnotetext{
una cosa es el método historiográfico del que se vale el historiador para conocer la historia, es decir, para hacer historiografía; y otra muy distinta es el control histórico que interesa al politólogo para hacer ciencia política (y al sociólogo para hacer sociología). El politólogo no es un historiador, y no hace historiografía. Le interesa únicamente el control histórico, vale decir un "tratamiento de la historia" apropiado para comprobar las leyes o para generar hipótesis generalizadoras (Sartori, 2002, p. 263).
}

Aquí se podría entrar en una discusión acerca de la idea de ciencia nomotética y su productividad social o con respecto a las fronteras disciplinares, o debatir el rol de las teorías de alcance intermedio y los estudios de caso que nutren a los estudios de la política. Sin embargo, estas discusiones nos desviarían del tema que nos interesa. Aquí vale la pena señalar que mientras que una parte de la ciencia política trataba de alejarse de la historia, la sociología hacía el camino inverso. Así, mientras Moore (1993) mostraba resultados fructíferos del uso de la historia, Sartori (2002, pp. 263-264) insistía en que el método histórico era desaconsejable, porque implicaba comparaciones que no incluían "paridad de condiciones", y porque los datos resultaban difíciles o imposibles de conseguir, lo que impedía asegurar el carácter plenamente científico de un estudio de este tipo. Pero incluso quienes, como Skocpol (1979, pp. xiv-xv), usaban con provecho a la historia parecían coincidir con la idea de que una cosa es lo que hacen los historiadores y otra los científicos sociales:

La tarea de quien realiza una comparación histórica no consiste en revelar nuevos datos acerca de aspectos particulares de los diversos periodos y lugares analizados en su estudio, sino, antes bien, en establecer el interés y la validez prima facie de un argumento general acerca de las regularidades causales, a través de los diversos casos históricos. El comparativista no tiene ni el tiempo ni (todas) las capacidades apropiadas para efectuar la investigación básica que necesariamente constituye, 
en gran medida, el fundamento sobre el cual se edifican los estudios de historia comparada. En cambio, debe concentrarse en escudriñar y revisar sistemáticamente las publicaciones de los especialistas que tratan de los asuntos definidos como importantes, por las consideraciones teóricas y por la lógica del análisis comparativo (...) [Así] la labor del comparativista sólo es posible después de que los especialistas han puesto a su disposición una amplia literatura básica. Sólo entonces puede tener esperanzas de encontrar al menos cierto material pertinente a cada tema, que debe investigarse según los dictados del argumento comparativo y explicativo que esté tratando de desarrollar.

Pero, uno podría preguntar qué sucede cuando la "amplia literatura básica" a la que se refiere Skocpol no está disponible, o si la misma presenta lagunas. ¿Y si se trata de un tema sobre el que los historiadores presentaron material y conclusiones contradictorias? ¿Deberíamos esperar a que los historiadores generen un consenso académico para solo luego de ello buscar regularidades? Además, ¿es efectivamente cierto que un científico social que no sea un historiador profesional carece de "el tiempo" y "las capacidades apropiadas" para hacer historia? Incluso, más allá de estos primeros interrogantes, ¿̇debería considerarse efectivamente a la ciencia política como una disciplina estrictamente comparativa? ¿No valdría la pena, acaso, ensayar un estudio propiamente histórico de ciertos fenómenos políticos? En este último sentido, hay quienes (por ejemplo, Kavanagh, 1991; de la Garza, 1993; Shapiro; Smith y Masoud, 2004; Morales, 2006; McLean, 2010) vienen insistiendo en el valor de un análisis que en lugar de usar a la historia como proveedora de datos para generar o validar hipótesis, la piensan como una dimensión del estudio de lo político (Pudal, 1989; Tilly y Wood, 2014).

Varios de los debates que repasamos ya carecen de sentido. Muchos de quienes hace algunas décadas se mostraban celosos de las fronteras y la metodología de la ciencia política han revisado sus certezas (así lo ha hizo el propio Sartori, 2005). Actualmente, métodos de trabajo que eran descartados, como el experimental, son reivindicados como fundamentales (Stoker, 2010) y la interdisciplinariedad, que alguna vez fue rechazada, no solo se da por sentada sino que se estimula (y no apenas con otras ciencias sociales, sino incluso con las ciencias naturales, Greaves y Grant, 2010). Por supuesto, hay quienes continúan tratando de colocar un cerco en derredor del estudio de lo político en nombre de ciertas concepciones de método o de metas, pero creo que están en franco retroceso.

\section{De la teoría AL PARTIDo}

En mis primeros trabajos sobre el neoliberalismo en Argentina usé herramientas de teoría política e historia intelectual para mostrar que el neoliberalismo no implicaba un estado ausente o bobo, que era mucho más diverso de lo que se solía considerar y que no era cierto que hubiese llegado de repente en 1989 o en 1976, afirmaciones que entonces estaban como instaladas en el discurso público y académico (Morresi, 2007, 2008, 2009). Pero también percibí que el abordaje resultaba un tanto limitado, porque los actores que estudiaba aparecían como receptores/traductores/difusores de ideas y solo de modo tangencial como sujetos con intereses y necesidades propios. Ante la ausencia relativa de trabajos de historia específicos sobre el neoliberalismo en Argentina, combiné la lectura de trabajos de historia política y social y emprendí tareas propias de un historiador: trabajo de archivo sobre diarios y revistas, pero también ensayos de divulgación, biografías, memorias, programas de estudios universitarios, correspondencia, grabaciones, así como entrevistas a algunos políticos e intelectuales a los que pude acceder. En este sentido, mi amistad con algunos historiadores dedicados a la historia reciente fue fundamental. En las trayectorias académicas, la fortuna juega un rol nada despreciable.

En 2010 estaba interesado en estudiar al partido Propuesta Republicana (PRO), porque varios de sus líderes tenían un discurso público cercano al neoliberalismo. Por otra parte, mi colega Gabriel Vommaro aquí la fortuna juega otra vez- también estaba interesado en el PRO como un caso para aplicar las ideas de socialización política sobre las que él venía trabajando. En principio me metí con un montón de literatura politológica sobre partidos políticos, pero esa reinmersión en la ciencia política me dejó con un sabor 
agridulce. Por un lado, refresqué conocimientos y me adentré en debates interesantes y actuales. Por el otro, como una parte importante de la bibliografía ponía el foco en el nivel sistémico, lo que más me interesaba a mí (las ideas de un partido, la forma en que esas ideas entraban en su agenda de propuestas, los debates acerca de esas ideas dentro y fuera de la organización, los alineamientos culturales de los miembros) quedaba un poco relegado. Fue en ese punto cuando, gracias a Vommaro conocí el trabajo de algunos politólogos que impulsaban un abordaje "societal" centrándose en la noción de "entornos partidarios" (Offerlé, 1987, 1999; Offerlé y Sawicki, 1996; Sawicki, 1997, 2011; Pudal, 1989; Schwartz, 1990) . En la óptica de estos autores los partidos no son entendidos como un ente cerrado y autónomo que refleja o reacciona ante los impulsos del sistema político o las condiciones sociales, sino como un conjunto de tramas (no siempre formalizadas ni ordenadas) que se asientan, se insertan y se nutren en una serie de mundos o esferas en los cuales los líderes y los cuadros dirigentes de un partido encuentran visiones de mundo, formatos para la acción y marcos de sentido que organizan las interacciones, las jerarquías internas y también la propia imagen de los partidos. Como sostiene Sawicki (2011, p. 43):

no se pueden disociar las organizaciones partidistas de su entorno, si se busca comprender cómo determinado partido logra implantarse en una sociedad dada. Aproximándose lo más posible al terreno, vale decir a nivel local, se constata fácilmente la porosidad de las fronteras entre los partidos políticos y su entorno social. Existe así un continuo de relaciones entre dirigentes, militantes, adherentes, simpatizantes y electores. En este sentido, el partido descansa en redes relacionales que se entrecruzan, que son basadas en valores o intereses compartidos, y alimentadas mediante interacciones en diversos lugares de sociabilidad más o menos formales: asociaciones, sindicatos, cooperativas, mutuales, cafés...

A partir de esta idea, junto con Vommaro estudiamos a PRO, con atención tanto a su enraizamiento social como a su construcción ético-política, mirando al mismo tiempo los espacios en que los que el PRO reclutaba militantes, cuadros políticos y se nutría de visiones del mundo, como los repertorios discursivos, ideológicos y morales que el partido iba adoptando y movilizando en una relación tensa con la tradición política en la que hundía sus raíces: la de la derecha política (Vommaro y Morresi, 2015; Vommaro; Morresi y Bellotti, 2015). En este sentido, la historia tenía un lugar fundamental en un doble sentido. Por un lado, precisábamos pensar las historias de los mundos y esferas en los que se asentaban las redes de PRO. Por el otro, debíamos poner las ideas políticas (y no solo políticas en sentido estricto) en relación con un espacio discursivo de posiciones históricamente determinado.

El objetivo de poner en relación las ideas del partido PRO con cierta tradición se encontró pronto con un limitante: los dirigentes de PRO se rehusaban a aceptar que las categorías derecha e izquierda tuviesen sentido. No es que apenas rechazasen el ser calificados como un partido de derecha, sino que impugnaban el propio concepto de derecha o izquierda. En ese momento, algunos colegas sugirieron que no correspondía usar categorías que no eran nativas, que debíamos abstenernos de usar términos como derecha, liberalismoconservador o neoliberalismo. No obstante, entendimos que lo interesante no era aceptar los modos de entenderse a sí mismos de los propios actores, sino encuadrar las visiones que se generaban en su entorno con las tradiciones políticas e incluso con las teorías sistemáticas de las que los protagonistas se nutrían consciente o inconscientemente. Así, no se trataba de imponer conceptos a los actores, sino de entender cómo se tensionaban entre la mirada de los que los rodeaban (que los señalaban como la derecha neoliberal o conservadora) y la suya propia (que sostenía que no lo eran), y por qué ese rechazo era fundamental para la imagen de sí mismos que querían proyectar no solo hacia afuera sino también hacia adentro. En este punto, siguiendo el planteo de Skinner (2002), la meta era entender sus "jugadas", y, así, comprender cómo se conformaba eso que podía identificarse como una "nueva" derecha argentina. Nueva por la forma en que combinaba distintas familias de derecha, que en Argentina habían sido divergentes, y porque tenía la vocación de acceder al poder por vía electoral (Morresi, 2012b, 2015). 


\section{EL CAMPO DE LA DERECHA Y LA HISTORIA}

La indagación sobre PRO, su entorno y las ideas de derecha, así como ciertos cuestionamientos que recibí por el uso de la categoría "derecha", me obligaron a repensar un poco las ideas que tenía acerca de la derecha política. Si bien tanto el abordaje de la historia social (Romero, 1970) como el de la teoría política (Bobbio, 1995) me parecía fundamentales, también me resultaban atractivos los aportes de la sociología política (Gibson, 1996; Lipset y Raab, 1981) y consideraba que los estudios politológicos destacaban factores relevantes (Colomer y Escatel, 2005; Wiesehomeier y Doyle, 2014). Así, en primera instancia, busqué combinar estos abordajes y definí a la derecha como una propuesta que da primacía a valores distintos de la igualdad y que defiende los intereses de ciertos sectores por sobre los de la mayoría, posicionándose reactivamente ante temas concretos en cada situación histórica.

Pese a que esta primera aproximación me pareció en su momento suficiente, luego percibí que no era del todo adecuada, que precisaba incorporar de un modo más claro la historia y las identidades de los actores considerados en plural. Hoy, creo que resulta más interesante pensar a la derecha y a la izquierda como cuerpos tridimensionales que funcionan de una forma similar a la de los campos magnéticos, como configuraciones relacionales dotadas de una gravedad específica que se impone a sus propios componentes e influye y repele a los elementos externos (Lewin, 1975; Bourdieu y Wacquant, 2005). Así, más que de "la derecha” o de "las derechas", conviene hablar del "campo de la derecha", como un espacio de interacción (de colaboración, competencia y conflicto) formado por actores y prácticas sobredeterminados por la gravedad del campo. En el caso de la derecha, esa gravedad está formada por procesos de identidad y comprensión que derivan de una serie acumulativa de rechazos concretos a innovaciones políticas, sociales, económicas, jurídicas o culturales de carácter igualitario o inclusivo que son percibidas como una desposesión (Morresi, 2019b).

Un cuestionamiento a este enfoque del campo de la derecha podría encontrarse en el potencialmente infinito número de rechazos a la inclusión y el carácter difuso de la "gravedad específica" del campo de la derecha. Sin embargo, justamente en este punto la historia (hecha por historiadores o por otros científicos sociales) tiene un rol fundamental para reducir ese conjunto de "rechazos concretos" a un conjunto de elementos teóricamente relevantes. Creo que por medio del trabajo historiográfico se puede identificar cuáles son los rechazos que forman parte de procesos de identidad/comprensión. En este sentido, interesan los rechazos que implican no solo una exclusión, sino que al mismo tiempo permiten que una idea (o conjunto de ideas) se erija en anatema de eso que ha sido excluido y se monumentalice como mito fundante y articulador, capaz de explicar acciones pasadas y justificar elecciones presentes y planes futuros. ${ }^{1}$ Asimismo, al observar las articulaciones de exclusión/mistificación también se torna más sencillo el ejercicio de identificar a las corrientes o familias de derecha, es decir, a los grupos de actores de un mismo campo, que comparten redes, ideas, espacios de socialización, memorias y lenguajes de una forma más estrecha (Nash, 1987; Rémond, 2007).

Si el enfoque del campo de la derecha resulta aceptable, corresponde estudiar a las derechas en contexto (lingüístico, pero también económico, social, cultural), es decir, de forma localizada, aunque ello no excluya, sino que más bien suponga la circulación de ideas y prácticas (Bertonha y Bohoslavsky, 2016). Por otra parte, el enfoque del campo implica también un estudio que incluya el análisis de las tradiciones y las memorias con las que el presente se entronca; un estudio del modo en que se recuperan, reactivan, resignifican y rearticulan ideas, lenguajes y prácticas en distintos ámbitos (partidos, ateneos, clubes, think tanks, diarios y revistas, centros de estudiantes, colegios profesionales, asociaciones civiles, ámbitos religiosos, cámaras empresariales, etc.). Es en esta instancia que la idea de "jugada" de Skinner cobra su pleno sentido: porque lo que interesa no es apenas qué es lo que se rechaza y qué es lo que se monumentaliza, sino cuáles son los argumentos, las palabras o los rituales que se emplean para ello. Aquí, es particularmente interesante encontrar "desviaciones" del canon teórico y del lenguaje corriente del campo, como por ejemplo, cuando Álvaro Alsogaray rechazaba de la Economía Social de Mercado el rol asignado a los sindicatos, o cuando Cecilia Pando reivindicó a los 
militares presos por su participación en el genocidio recurriendo a la misma liturgia que los organismos de derechos humanos (Morresi, 2012a; Morresi, 2019a).

Una vez identificados los ámbitos que nos interesan hay que concentrarse en el estudio de las ideas y prácticas de los actores que los transitan. Idealmente deberíamos hacer un trabajo en profundidad de cada una de esos mundos, pero eso no siempre es factible, sobre todo cuando no se encuentran activos o no podemos acceder a su funcionamiento interno. En estos casos podemos buscar material (académico o no) sobre esos espacios, y producciones textuales o testimonios que nos ayudaren a recrear su organización, su funcionamiento y los discursos y las ideas que allí circulan o circularon.

Sobre las ideas y discursos, creo necesario hacer algunos apuntes más. En primer lugar, me parece importante destacar que hay que mirar a las ideas de derecha bajo la mejor luz posible. En muchos de los estudios sobre las derechas (incluso en algunos muy buenos) las ideas derechistas son expuestas como si fuesen apenas excusas ad hoc para impulsar un interés económico; mantos para ocultar una pura voluntad de poder o el intento de racionalizar lo irracional. Creo que eso es un error y que debemos intentar leer a las ideas de derecha como orientadas a un "bien común", que podemos no compartir, pero que precisamos comprender. En segundo lugar, a la hora de estudiar las ideas del campo de la derecha continúa siendo interesante considerar como eje al canon de la teoría política. Gracias a él se pueden identificar grandes corrientes de pensamiento (por ejemplo, liberalismo o conservadurismo), algunas líneas más tenues (como liberalismoconservador o neoliberalismo), y otras clasificaciones que resultan útiles (como positivismo o romanticismo). En cierto sentido, el canon de la teoría política es un mapa que permite una mejor comprensión de los datos. Finalmente, concentrarse en las ideas y discursos facilita distinguir y reconstruir los repertorios de cada familia de derecha (Rémond, 2007) y ver el modo en que el campo se despliega (cuáles actores con cuáles discursos son hegemónicos, los modos en que los actores del mismo campo se diferencian, interactúan y disputan entre sí).

El enfoque del campo de la derecha puede engarzar dos temporalidades distintas. Por un lado, una temporalidad "corta", en la que se privilegian las trayectorias de los actores que se van a estudiar (por ejemplo, la de Álvaro Alsogaray o la de Martínez de Hoz). Por el otro, a una temporalidad “larga”, que da cuenta de la historia de las ideas que esos mismos actores retoman y recrean. No se trata apenas de decir X toma la idea del autor $Y$, sino de tratar de indagar cómo, a través de qué mecanismos y con qué fines actúa $X$ al tomar esa idea de Y. Para poner un ejemplo, si un político argentino reivindica la idea de mercado formulada por Adam Smith, no está leyendo solo a Smith, sino leyéndolo a través de un determinado prisma interpretativo, como, por ejemplo, la particular visión de Friedman (1976) o la que estaba presente en las discusiones de los organismos internacionales de crédito (Chwieroth, 2010). A su vez, no está leyendo a ese autor o movilizando sus ideas por azar, sino porque tiene intereses inmediatos, quizás impulsar una determinada política u oponerse a una iniciativa concreta. Entonces, lo que interesa no es apuntar que un determinado político tenga una interpretación sesgada de la idea de mercado de Adam Smith, sino entender por qué ese actor precisa o entiende que es relevante invocar a Adam Smith, cuáles de sus ideas toma en cuenta y cuáles no, con qué lecturas de Smith se entronca su propia visión, cuáles son sus intereses inmediatos (políticos, económicos, institucionales), en qué sentido el uso de Adam Smith lo ayuda a impulsarlos, qué aliados tiene a la hora de interpretar de ese modo a Smith, el eco que obtiene su interpretación y si consigue que la misma se torne parte del sentido común (de su subcampo, del campo de la derecha o incluso de todo el espacio político).

Ya para ir finalizando, creo que el abordaje del campo de la derecha pone en claro la amplitud y la porosidad de eso que suele denominarse sistema político. Aquí no solo importan los partidos y grupos políticos de derecha en un sentido restringido, sino los diversos mundos en donde se asientan y de los cuales se nutren (de cuadros, de militantes, de ideas, de prácticas). En este sentido, los espacios intelectuales y educativos y los empresariales y profesionales son particularmente importantes. Sin embargo, la lista de esferas es siempre incompleta y hay espacios que aún deben ser estudiados (el recreativo, por ejemplo). La afirmación de que la política "está en todas partes" puede ser una trivialidad, pero no por ello deja ser cierta. 


\section{CODA}

A lo largo de estas páginas, en las que se entremezclaron los argumentos con la memoria personal y académica, traté de reflejar parte del intercambio de ideas que se dio en el encuentro Usos de la historia para investigar problemas sociales y explicar cómo llegué a pensar en estudiar a la derecha como un campo, por qué ese enfoque me parece fructífero y destacar cuál es el rol de la historia y la historiografía en ese abordaje.

Sin embargo, antes de poner el punto final, quisiera dedicar unas pocas líneas a recuperar un temaque estaba en el cuestionario que disparó el debate: el papel de la rigurosidad en la investigación. A este respecto, entiendo que una investigación rigurosa debe cumplir con tres principios generales: ser tan exhaustiva como sea posible en un determinado momento (por supuesto, hay cuestiones que nos exceden, como la imposibilidad de acceder a algunas fuentes o la falta de recursos para realizar determinados estudios); ser clara y honesta en sus alcances (con esto quiero aludir a la necesidad de dejar asentados no solo los límites del estudio realizado, sino también aquellos que se derivan del uso de una cierta metodología y un determinado marco teórico) y ser contestable (en el sentido de estar estructurada y presentada de un modo tal que permita la crítica y el diálogo con otros abordajes). Creo que la última idea merece ser enfatizada, porque, además de rigurosas, las investigaciones deberían estar enmarcadas en conversaciones amplias y fructíferas, que trasciendan las fronteras disciplinares y no se circunscriban a los seguidores de un determinado marco teórico o enfoque metodológico.

\section{ReFERENCIAS}

Almond, G. A. (1996). Political Science: The History of the Discipline. En R. E. Goodin y H.-D. Klingemann (Eds.), A New Handbook of Political Science (1) (pp. 50-96). Oxford: Oxford University Press.

Bartolini, S. (1993). On Time and Comparative Research. Journal of Theoretical Politics, 5(2), 131-167. http://dx.do i.org/10.1177/0951692893005002001.

Bartolini, S. (1994). Metodología de la investigación política. En G. Pasquino, S. Bartolini, A. Panebianco y L. Morlino (Eds.), Manual de ciencia política (pp. 39-77). Madrid: Alianza.

Bertonha, J. F., y Bohoslavsky, E. (2016). Las derechas sudamericanas: trayectorias, miradas y circulación. En J. F. Bertonha y E. Bohoslavsky (Eds.), Circule por la derecha: percepciones, redes y contactos entre las derechas sudamericanas, 1917-1973 (pp. 9-18). Los Polvorines: UNGS.

Bevir, M. (1999). The logic of the history of ideas. New York: Cambridge University Press.

Bobbio, N. (1995). Derecha e izquierda: razones y significados de una distinción política (A. Picberlione, Trad.). Buenos Aires: Taurus.

Bourdieu, P. y Wacquant, L. (2005). Una invitación a la sociología comprensiva. Buenos Aires: Siglo XXI.

Chwieroth, J. M. (2010). Capital ideas: the IMF and the rise of financial liberalization. Princeton: Princeton University Press.

Colomer, J. M. (2006). La ciencia política va hacia adelante (por meandros tortuosos). Un comentario a Giovanni Sartori. Revista Española de Ciencia Política, 9(2), 41-45.

Colomer, J. M., y Escatel, L. E. (2005). La dimensión izquierda-derecha en América Latina. Desarrollo Económico, 45(177), 123-136. http://www.jstor.org/stable/3655894.

de la Garza, L. A. (1993). Historia y Ciencia Política. Relaciones - Estudios de Historia y Sociedad, XIV(53), 35-44.

Dosse, F. (2007). La marcha de las ideas: historia de los intelectuales, historia intelectual (R. Tomás, Trad.). Valencia: Universitat de València.

Friedman, M. (1976). Adam Smith's relevance for 1976. Chicago Booth Review, (Winter 1976). https://review.chica gobooth.edu/economics/archive/adam-smiths-relevance-1976. 
Gibson, E. L. (1996). Class and conservative parties: Argentina in comparative perspective. Baltimore: Johns Hopkins University Press.

Greaves, J., y Grant, W. (2010). Crossing the Interdisciplinary Divide: Political Science and Biological Science. Political Studies, 58(2), 320-339.

Kavanagh, D. (1991). Why Political Science Needs History. Political Studies, 39(3), 479-495.

Lewin, K. (1975). Field theory in social science: selected theoretical papers. Westport, Conn.: Greenwood Press.

Lipset, S. M., y Raab, E. (1981). La política de la sinrazón: el extremismo de derecha en los Estados Unidos 1790-1970 (J. J. Utrilla, Trad.). México, DF: Fondo de Cultura Económica.

Machiavelli, N. (1969). Carta de Maquiavelo a Francesco Vettori, embajador de la República Florentina en Roma (1513), traducida por Aliíde Foppa. Revista de la Universidad de México, (9), 30-32.

McLean, I. (2010). Political Science and History: Friends and Neighbours. Political Studies, 58(2), 354-367.

Mirowski, P. (2014). Never let a serious crisis go to waste: how neoliberalism survived the financial meltdown. Londres: Verso.

Mirowski, P., y Plehwe, D. (Eds.). (2009). The road from Mont Pelerin: the making of the neoliberal thought collective. Cambridge, Mass.: Harvard University Press.

Moore, B. (1993). Social origins of dictatorship and democracy: lord and peasant in the making of the modern world. Boston: Beacon Press.

Morales, R. (2006). ¿Qué lugar ocupa la historia en la ciencia política? In V. Alarcón Olguín (Ed.), Metodologías para el análisis politico: enfoques, procesos e instituciones (pp. 121-136). México, D. F.: Universidad Autónoma Metropolitana.

Morresi, S. D. (1997). La libertad y la necesidad de hacer política. I como Icaro, 1(2), 32-38.

Morresi, S. D. (2002). Nozick e o liberalismo fora do esquadro. Lua Nova, (55-56), 285-296.

Morresi, S. D. (2006). O liberalismo desenquadrado. Uma crítica às leituras neoliberais do liberalismo clássico (Tesis de doctorado). Faculdade de Filosofia e Letras, Universidade de São Paulo, São Paulo.

Morresi, S. D. (2007). ¿Más allá del neoliberalismo? Estado y neoliberalismo en los años noventa. En E. Rinesi, G. Nardacchione y G. Vommaro (Eds.), Las lentes de Victor Hugo. Transformaciones políticas y desafíos teóricos en la Argentina reciente (pp. 117-150). Buenos Aires: Prometeo.

Morresi, S. D. (2008). La nueva derecha argentina y la democracia sin política. Buenos Aires: Biblioteca Nacional UNGS.

Morresi, S. D. (2009). Neoliberales antes del Neoliberalismo. En G. Soprano y S. Frederic (Eds.), Politica y variaciones de escalas en el análisis de la Argentina (pp. 321-350). Buenos Aires: Prometeo.

Morresi, S. D. (2011). Un esquema analítico para el estudio de las ideas de derecha en Argentina (1955-1983). En E. Bohoslavsky (Ed.), Actas del Taller de Discusión sobre las Derechas en el Cono Sur, siglo XX (pp. 23-41). Los Polvorines: UNGS.

Morresi, S. D. (2012a). La protesta de la derecha argentina después de 2001. Ponencia presentada en Panel: Morfologías de la acción colectiva después de 2001, Instituto Gino Germani, Universidad de Buenos Aires, Buenos Aires.

Morresi, S. D. (2012b). Right and Center-right in contemporary Argentina: The PRO party case. Ponencia presentada en XXII World Congress of Political Science, IPSA, Madrid.

Morresi, S. D. (2015). “Acá somos todos democráticos”. El PRO y las relaciones entre la derecha y la democracia en la Argentina. En G. Vommaro y S. D. Morresi (Eds.), "Hagamos equipo". PRO y la construcción de la nueva derecha argentina (pp. 163-201). Buenos Aires: Prometeo.

Morresi, S. D. (2018). Cara al sol: el pensamiento de las derechas políticas durante el siglo XX. En R. Álvarez (Ed.), La Filosofía en el siglo XX (pp. 59-102). Buenos Aires: Prometeo.

Morresi, S. D. (2019a). "Reconocer lo actuado”. El liberalismo-conservador y sus miradas sobre la dictadura y la violencia (1982-1989). Revista de Historia Americana y Argentina, 54(2), 207-238. http://revistas.uncu.edu.ar/ ojs/index.php/revihistoriargenyame/article/view/2830/2024. 
Morresi, S. D. (2019b). As direitas argentinas e a democracia: ditadura e pos-ditadura. En R. Patto Sá Motta, E. Bohoslavsky y S. Boisard (Eds.), Pensar as direitas na América Latina (pp. 37-55). Belo Horizonte: Alameda.

Nash, G. H. (1987). La rebelión conservadora en los Estados Unidos. Buenos Aires: Grupo Editor Latinoamericano.

Offerlé, M. (1987). Les partis politiques. Paris: Presses Universitaires de France.

Offerlé, M. (1999). La profession politique: XIXe-XXe siècles. Paris: Belin.

Offerlé, M., y Sawicki, F. (Eds.). (1996). Entrées en politique: apprentissages et savoir-faire. Paris: L'Harmattan.

Plehwe, D. (2018). Neoliberal Thought Collectives: Integrating Social Science and Intellectual History. En D. Cahill, M. Cooper, M. Konings y D. Primrose (Eds.), The Sage handbook of neoliberalism (pp. 85-97). Thousand Oaks: SAGE.

Plehwe, D., Walpen, B., \& Neunhöffer, G. (Eds.). (2005). Neoliberal hegemony: a global critique (Vol. 18). Milton Park, Abingdon \& New York: Routledge.

Pocock, J. G. A. (2011). Pensamiento politico e historia: ensayos sobre teoria y método (S. Chaparro, Trad.). Madrid: Akal.

Pudal, B. (1989). Prendre parti: pour une sociologie historique du PCF. Paris: Presses de la Fondation nationale des sciences politiques.

Rémond, R. (2007). Les droites aujourd'hui. Paris: Éd. L. Audibert.

Romero, J. L. (1970). El pensamiento politico de la derecha latinoamericana. Buenos Aires: Paidós.

Sartori, G. (1974). Philosophy, Theory and Science of Politics. Political Theory, 2(2), 133-162.

Sartori, G. (2002). La politica: lógica y método en las ciencias sociales (M. Lara, Trad. $3^{\circ}$ ed.). México, DF: Fondo de Cultura Económica.

Sartori, G. (2005). ¿Hacia dónde va la ciencia política? Revista Española de Ciencia Política, (12), 9-13.

Sawicki, F. (1997). Les réseaux du Parti socialiste: sociologie d'un milieu partisan. Paris: Belin.

Sawicki, F. (2011). Para una sociología de los entornos y de las redes partidistas. Revista de Sociología, (25), 37-53.

Schwartz, M. A. (1990). The party network: the robust organization of Illinois republicans. Madison, Wis.: University of Wisconsin Press.

Shapiro, I., Smith, R. M., \& Masoud, T. E. (Eds.) (2004). Problems and Methods in the Study of Politics. Cambridge: Cambridge University Press.

Skinner, Q. (2002). Visions of politics. Regarding method. Cambridge: Cambridge University Press.

Skocpol, T. (1979). States and social revolutions: a comparative analysis of France, Russia, and China. Cambridge: Cambridge University Press.

Stoker, G. (2010). Exploring the Promise of Experimentation in Political Science: Micro-Foundational Insights and Policy Relevance. Political Studies, 58(2), 300-319.

Tilly, C., y Wood, L. J. (2014). Los movimientos sociales, 1768-2008. Desde sus orígenes a facebook (F. Esteve, Trad.). Barcelona: Crítica.

Vommaro, G., y Morresi, S. D. (Eds.). (2015). "Hagamos equipo". PRO y la construcción de la nueva derecha argentina. Los Polvorines: Universidad Nacional de General Sarmiento.

Vommaro, G.; Morresi, S. D. y Bellotti, A. (2015). Mundo PRO. Anatomía de un partido fabricado para ganar. Buenos Aires: Planeta.

Wiesehomeier, N., \& Doyle, D. (2014). Profiling the Electorate: Ideology and Attitudes of Rightwing Voters. En J. P. Luna y C. Rovira Kaltwasser (Eds.), The Resilience of the Latin American Right (pp. 48-72). Baltimore: Johns Hopkins University Press.

\section{Notas}

1 Así, para el caso del liberalismo-conservador argentino, el concepto excluido fue populismo, mientras que la idea de república se transformó en mito articulador (Morresi, 2011). 